\title{
Surfactant use in late preterm infants: a survey among Belgian neonatologists
}

\author{
L. Cornette ${ }^{1}$ (I) $\cdot$ A. Mulder ${ }^{2} \cdot$ A. Debeer ${ }^{3} \cdot$ G. Malfilâtre ${ }^{4} \cdot$ V. Rigo ${ }^{5} \cdot$ F. Cools ${ }^{6} \cdot$ O. Danhaive $^{7,8}$ \\ Received: 30 July 2020 / Revised: 5 September 2020 / Accepted: 8 September 2020 / Published online: 24 September 2020 \\ (C) Springer-Verlag GmbH Germany, part of Springer Nature 2020
}

\begin{abstract}
Specific recommendations on surfactant administration in late preterm (LPT) infants with pulmonary disease are lacking. We performed an online-based, nationwide survey amongst all $(n=102)$ Belgian neonatologists to identify the use of surfactant in LPT infants suffering from several respiratory pathologies. The survey used clearly defined clinical cases and resulted in a $86 \%$ response rate. Neonatologists adhere to the $200 \mathrm{mg} / \mathrm{kg}$ initial surfactant dosing scheme. Surfactant is widely used in respiratory distress syndrome $(70.1 \%)$, but there is less unanimity on its use in meconium aspiration syndrome (58.0\%), transient tachypnoea of the newborn (30.6\%), congenital pneumonia $(27.2 \%)$ and congenital diaphragmatic hernia $(8.6 \%)$. Respondents adhere to the European guideline of a timely referral to a newborn intensive care unit (non-invasive ventilation and $\mathrm{FiO}_{2}>0.30$ at $12 \mathrm{~h}$ of age), in order to minimise the risk of deterioration.

Conclusion: We demonstrate a wide variety in the use of surfactant within LPT infants. The majority of Belgian neonatologists therefore urge for an investment in multi-centre trials on surfactant administration in LPT infants, in order to create an evidencebased practice as well as to reduce the strain on health care budgets.
\end{abstract}

Trial registration: https://clinicaltrials.gov

\section{What is Known:}

- Any late preterm (LPT) infant with respiratory distress needs a timely referral to a neonatal intensive care unit in case of non-invasive ventilation and $\mathrm{FiO}_{2}>0.30$ at $12 \mathrm{~h}$ of life, in order to minimise the risk of acute deterioration as well as chronic lung disease.

- Any modest increase in morbidity in the sizeable group of LPT infants exerts a significant strain on health care budgets.

What is New:

- We report the attitudes and opinions of Belgian neonatologists about the use of surfactant in LPT infants suffering from several respiratory diseases.

- Our survey demonstrates a significant variability in practice between neonatologists during treatment of respiratory pathologies in LPT infants. This highlights an urgent need for univocal therapeutic lines.

Communicated by Daniele De Luca

\section{Cornette}

luc.cornette@azsintjan.be

A. Mulder

antonius.mulder@uza.be

A. Debeer

anne.debeer@uzleuven.be

G. Malfilâtre

malfilatre.genevieve@gmail.com

V. Rigo

vincent.rigo@chu.ulg.ac.be

F. Cools

filip.cools@uzbrussel.be

O. Danhaive

olivier.danhaive@uclouvain.be
1 AZ St-Jan Brugge, Ruddershove 10, 8000 Bruges, Belgium

2 UZ Antwerpen, Wilrijkstraat 10, 2650 Edegem, Belgium

3 UZ Leuven, Herestraat 49, 3000 Leuven, Belgium

4 CHU Tivoli, Avenue Max Buset 34, 7100 La Louvière, Belgium

5 CHU de Liège, Boulevard du Douzième de Ligne 1, 4000 Liège, Belgium

6 UZ Brussel, Avenue du Laerbeek 101, 1090 Jette, Belgium

7 UC Louvain, Place de l'Université 1, 1348 Ottignies-Louvain-la-Neuve, Belgium

8 Department of Pediatrics, Benioff Children's Hospital, University of California San Francisco, San Francisco, CA, USA 
Keywords Late preterm infant $\cdot$ Surfactant therapy $\cdot$ Respiratory distress syndrome $\cdot$ Nationwide survey

$\begin{array}{ll}\text { Abbreviations } \\ \text { CDH } & \text { Congenital diaphragmatic hernia } \\ \text { CP } & \text { Congenital pneumonia } \\ \text { CPAP } & \text { Continuous positive airway pressure } \\ \text { GA } & \text { Gestational age } \\ \text { iNO } & \text { Inhaled nitric oxygen } \\ \text { INSURE } & \text { Intubation, surfactant administration, extubation } \\ \text { LISA } & \text { Less invasive surfactant administration } \\ \text { LPT } & \text { Late preterm } \\ \text { MAS } & \text { Meconium aspiration syndrome } \\ \text { NICU } & \text { Newborn intensive care unit } \\ \text { PPHN } & \text { Persistent pulmonary hypertension of the } \\ & \text { newborn } \\ \text { nRDS } & \text { Neonatal respiratory distress syndrome } \\ \text { TTN } & \text { Transient tachypnoea of the newborn }\end{array}$

\section{Introduction}

Late preterm (LPT) infants (i.e. infants born between 34 and $36+6$ weeks GA) account for up to $75 \%$ of all preterm births. Historically these babies were classified as 'near term', since they have weights similar to term infants at birth, making them appear deceivingly healthy $[1,2]$. However, LPT infants have structurally immature lungs and may have a suboptimal surfactant production. Compared with infants born at term, LPT infants are more likely to suffer poorer short-term outcomes such as neonatal respiratory distress syndrome (nRDS) (relative risk [RR], 17.3), intraventricular haemorrhage (RR, 4.9) and death $<28$ days (RR, 5.9) [3].

The fourth update of the European Consensus Guidelines on the Management of Respiratory Distress Syndrome summarises the evidence regarding indications, administration and outcomes for surfactant replacement therapy [4]. Within the group of LPT infants, many simultaneously present characteristics of nRDS, as well as transient tachypnoea of the newborn (TTN) and persistent pulmonary hypertension of the newborn (PPHN) in the course of the same respiratory disease. Such complicates any neonatologist's decision whether or not to give surfactant. With respect to TTN or PPHN in the LPT infant, neonatologists base their decisions to administer surfactant on small single-centre studies, as there are no large prospectively organised trials available [5]. Also, a distinction between surfactant deficiency nRDS and lung inflammation/infection due to chorio-amnionitis is sometimes difficult to make during routine clinical practice [6].

Hence, although surfactant is commonly used in the management of respiratory failure in LPT infants, many questions remain unanswered:
- What is the optimal timing and dosage of surfactant administration in LPT infants?

- What is the correct $\mathrm{FiO}_{2}$ threshold to administer surfactant?

- Should we administer surfactant in the context of TTN, meconium aspiration syndrome (MAS), congenital pneumonia $(\mathrm{CP})$ or congenital diaphragmatic hernia $(\mathrm{CDH})$ ?

We therefore need to better understand why and how surfactant is used for different respiratory pathologies seen in LPT infants. In order to create a Belgian consensus statement on the use of surfactant within these infants, we needed a clear idea of individual practices across all newborn intensive care units (NICUs). As such, we conducted a nationwide survey, i.e. a collection of data in a standardised way.

\section{Materials and methods}

This survey research has been conducted based on a good practice checklist [7]. Seven Belgian neonatologists with a specific interest in neonatal respiratory diseases were selected based on their previous clinical and scientific experience. Consensus on the development of 5 cases as well as several survey questions was obtained during two face-to-face meetings in which a mini-Delphi technique was used, i.e. an interactive and structured discussion forum. The meetings were expanded with email exchange, i.e. a process of continuous interaction, enabling the seven panellists to exchange their final comments. LC acted as the facilitator (sending out questions, collecting responses, identifying common and conflicting viewpoints) to achieve consensus and to converge to the final questionnaire, i.e. an online survey tool (SurveyMonkey, Portland, OR).

The survey was then conducted using a non-random sampling technique, i.e. the structured questionnaire was sent to the unique group of Belgian neonatologists $(n=102)$, working in one of 19 NICUs, covering 118,000 deliveries per year. The survey was not sent to junior doctors in training, but only to certified neonatologists that are active within a clinical setting. All neonatologists were invited by email (neonatology.belgium@gmail.com) to participate. One reminder was sent 2 months after the initial invitation, in order to increase the response rate. The survey has been approved by the Ethical Comité of AZ St-Jan Bruges-Ostend AV. All participants had to read through a covering letter explaining the rationale of the survey, as well as what will happen with the information provided. Prior to starting the survey, they needed to tick a box in order to consent. The study was registered onto https://clinicaltrials.gov. 
Each person completed the survey in a semi-anonymous way: no personal names were asked. However, in order to ensure there were a sufficiently high number of neonatologists responding from each unit, we asked for the hospital's name each person was working at.

Five written clinical cases were presented to the neonatologists (Table 1), together with the same set of structured questions for each clinical case. The survey ended with openended questions regarding patient selection and experiences of surfactant efficacy within this LPT patient group.

Data analysis is descriptive. Categorical variables are presented in absolute numbers and percentages.

Table 1 Description of 5 clinical cases

Case 1

A 34-week 2/7 GA infant is born (2.750 g); the mother is GBS negative. There is no PPROM. The infant is tachypnoeic at birth $(80 / \mathrm{min})$ and is in need of $\mathrm{FiO}_{2}$ 0.25. IV access is obtained, blood culture taken and IV antibiotics are started. The infant is transferred to the NICU. At the age of $1 \mathrm{~h}$, the chest X-ray shows an air bronchogram. The infant remains tachypnoeic and needs $\mathrm{FiO}_{2} 0.35$. nCPAP is started. At the age of $4 \mathrm{~h}$, the infant still needs $\mathrm{FiO}_{2} 0.38$

Case 2

A 38-week GA infant is born $(3.800 \mathrm{~g})$; the mother is GBS negative. The patient presents with foetal tachycardia after delivery by emergency $\mathrm{C}-$ Section. Amniotic fluid is meconium stained. The infant needs intubation and ventilation at the age of $1 \mathrm{~min}$. At the age of $3 \mathrm{~h}$, the infant saturates $86 \%$ with invasive ventilation (SIMV), pressures $24 / 5 \mathrm{~cm}$ $\mathrm{H}_{2} \mathrm{O}$, frequency $45 / \mathrm{min}, \mathrm{FiO}_{2} 0.60$. Chest X-ray is patchy. The following parameters are available on an arterial blood gas: $\mathrm{pH} 7.10$, $\mathrm{PaCO}_{2} 55 \mathrm{mmHg}$ and $\mathrm{PaO}_{2} 45 \mathrm{mmHg}$. Cardiac ultrasound demonstrates evidence of pulmonary hypertension.

Case 3

A 36-week1/7 GA infant is born (2.900 g) after an urgent Caesarean section within the context of acute maternal blood loss (abruption of the placenta). The infant is born in good condition, but needs $\mathrm{FiO}_{2} 0.30$ during the first golden minutes of life. Intravenous access is obtained, blood culture taken. The infant is transferred to the NICU with nCPAP and $\mathrm{FiO}_{2} 0.30$. At the age of $2 \mathrm{~h}$, the chest X-ray shows extra fluid in the fissures separating the long lobes. At the age of $4 \mathrm{~h}$, having inserted umbilical lines, the infant remains tachypnoeic and needs $\mathrm{FiO}_{2} 0.40$.

Case 4

A 35-week GA infant is born ( $2.750 \mathrm{~g})$; the mother is GBS positive. There is no PPROM. The infant needs 5 insufflations, remains tachypnoeic $(90 / \mathrm{min})$ and is in need of $\mathrm{FiO}_{2}$ 0.35. IV access is obtained, blood culture taken and IV antibiotics are started. The infant is transferred with nCPAP to the NICU. At the age of $2 \mathrm{~h}$, chest X-ray shows patchy lung fields. CRP level is $42 \mathrm{mg} / \mathrm{L}$ on D0. The infant remains tachypnoeic and needs $\mathrm{FiO}_{2}$ between 0.30 and 0.40 .

Case 5

A 36-week GA infant is born $(2.900 \mathrm{~g})$; the mother is GBS negative. The infant presents with severe respiratory distress at birth, requiring intubation and ventilation. A chest X-ray demonstrates the presence of bowel in the left hemi-thorax. At the age of $3 \mathrm{~h}$, SIMV ventilation is switched to high-frequency oscillation ventilation with the following settings: mean airway pressure $11 \mathrm{~cm} \mathrm{H}_{2} \mathrm{O}$, Delta $\mathrm{P} 28 \mathrm{~cm} \mathrm{H}_{2} \mathrm{O}, \mathrm{FiO}_{2}$ 0.45. An arterial blood gas shows $\mathrm{pH} 7.15, \mathrm{PaCO}_{2} 52 \mathrm{mmHg}, \mathrm{PaO}_{2} 49$ $\mathrm{mmHg}$. The infant continues to saturate at best $82 \%$.

\section{Results}

All participants were given a 6 months' time window to respond to the survey (September 2019 to February 2020). Two reminders (month 3 and month 5) were sent.

Responses were received from $n=90 / 102$ neonatologists ( $n=65$ females, $n=25$ males). All Belgian NICUs were represented (response rate varying between 33 and $100 \%$ per unit). Two participants did not fully complete the survey; hence, their responses were excluded. We performed all analyses on $n=88 / 102$ responses, i.e. a response rate of $86 \%$.

Clinical experience is spread across four age groups as follows (the number of years one has been working as a neonatologist): $40.2 \%$ ( $<$ or $=10$ years), $37.9 \%$ (11-20 years), $18.4 \%$ ( $21-30$ years), $3.4 \%$ ( $31-40$ years). All units exclusively use poractant alfa.

\section{Administration of surfactant (case 1-case 5)}

Figure 1 indicates the $\%$ of respondents that will administer (or not) surfactant to the 5 clinical cases. Surfactant in LPT infants is mostly given for nRDS (70.1\%) and MAS (58.0\%). In TTN and CP, many respondents prefer not to give surfactant, quoting reasons such as preference to optimise the infant's position and non-invasive ventilator support, together with cardiac ultrasound to exclude PPHN. In CDH, surfactant is rarely used, as most respondents remain indecisive. Respondents prefer the administration of iNO, as well as an optimisation of blood pressure management. Those respondents that give surfactant in CDH note clearly that it is mostly given as a 'rescue' therapy, especially when ventilation remains difficult.

\section{Method of administration of surfactant (case 1-case 4 )}

Regarding the method of surfactant administration, the majority prefers the less invasive approach in the case of RDS, TTN and CP, i.e. less invasive surfactant administration (LISA) or intubation, surfactant administration, extubation (INSURE) (Fig. 2). It is however worth noting that, when an LPT infant needs a second dose of surfactant in these cases, $45.6 \%$ of the respondents administer the second dose after intubation and ventilation only. In the case of MAS, the majority of the respondents $(n=88.0 \%)$ will preferentially administer surfactant using intubation and ventilation.

\section{Threshold $\mathrm{FiO}_{2}$ to administer surfactant (cases 1, 2 and 4 )}

In case 1 (nRDS), threshold $\mathrm{FiO}_{2}$ for transfer to a high care neonatal centre that can provide the appropriate level of care (and thus the administration of surfactant) is $\geq 0.30$ in $76.3 \%$ 
Fig. 1 Administration of surfactant (case 1-case 5). Expressed in \% of neonatologists. 'Other' refers to neonatologists that are indecisive and may include careful blood pressure management or a trial of Inhaled nitric oxygen (iNO), prior to 'possibly' reverting to the administration of surfactant (as a final rescue). nRDS, neonatal respiratory distress; MAS, meconium aspiration syndrome; TTN, transient tachypnoea of the newborn; $\mathrm{CP}$, congenital pneumonia; $\mathrm{CDH}$, congenital diaphragmatic hernia

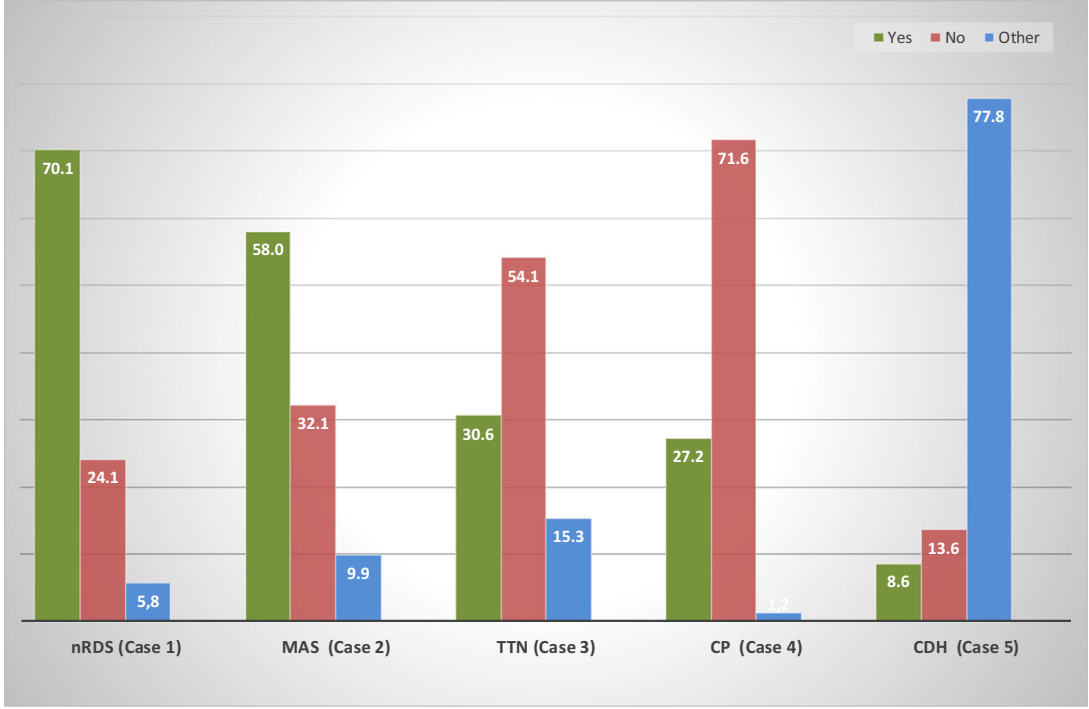

( $n=54)$ and $\geq 0.40$ in $23.7 \%(n=15)$ (Fig. 3). Of those respondents who consider surfactant administration in case 1 (i.e. below 24 h of age), only $n=38$ still consider surfactant when the infant would be between 24 and $48 \mathrm{~h}$ of age. The $24.1 \%$ of neonatologists $(n=21)$ who would not give surfactant to case 1 were asked at which $\mathrm{FiO}_{2}$ they would give surfactant if the infant further deteriorates: the majority $(n=$ 17) will give surfactant as soon as $\mathrm{FiO}_{2} \geq 0.40$ is needed, whilst the other $n=4$ will wait until an $\mathrm{FiO}_{2} \geq 0.50$ is needed. The majority (66\%) of the latter group prefers to use a clinical nRDS score in order to help them to decide.

$32.1 \%$ of the respondents $(n=29)$ will not give surfactant in MAS whereas $n=9$ envisages to do so as soon as $\mathrm{FiO}_{2} \geq$ $0.50 .54 .1 \%$ of the respondents $(n=47)$ will not give surfactant in TTN whereas $n=21$ is prepared to do so as soon as $\mathrm{FiO}_{2} \geq 0.40$. A larger group of $n=25$ respondents prefers to even wait until $\mathrm{FiO}_{2} \geq 0.40 .71 .6 \%$ of the respondents $(n=63)$ will not administer surfactant in CP, and two third (65.5\%) within that group would not consider surfactant even in the case of $\mathrm{FiO}_{2} \geq 0.40$.

\section{Dosing of surfactant (case 1-case 4 )}

In nRDS, the majority (95\%) agree with a theoretical dose of $200 \mathrm{mg} / \mathrm{kg}$. However, depending on the weight of the infant, $51 \%$ of the respondents admit they prefer to downscale the dosage of poractant alfa to $160 \mathrm{mg} / \mathrm{kg}$ (e.g. $480 \mathrm{mg}$ in case 1 or 2 vials of $240 \mathrm{mg}$ ) whilst in reality, $44 \%$ will administer the full $200 \mathrm{mg} / \mathrm{kg}$ (e.g. $600 \mathrm{mg}$ in case 1 or 2 vials of $240 \mathrm{mg}$ and 1 vial of $120 \mathrm{mg}$ ). The majority of neonatologists accept a difference of maximally $50 \mathrm{mg} / \mathrm{kg}$ between the recommended $(200 \mathrm{mg} / \mathrm{kg}$ ) and practical dosages $(150-200 \mathrm{mg} / \mathrm{kg}$ ).

In TTN, the majority (92\%) of the respondents agree with a theoretical dose of $200 \mathrm{mg} / \mathrm{kg}$ and accept a difference of
Fig. 2 Method of surfactant administration (case 1-case 4) Expressed in \% of neonatologists. LISA, less invasive surfactant administration; INSURE, intubation, surfactant administration, extubation; nRDS, neonatal respiratory distress syndrome; MAS, meconium aspiration syndrome; TTN, transient tachypnoea of the newborn; CP, congenital pneumonia

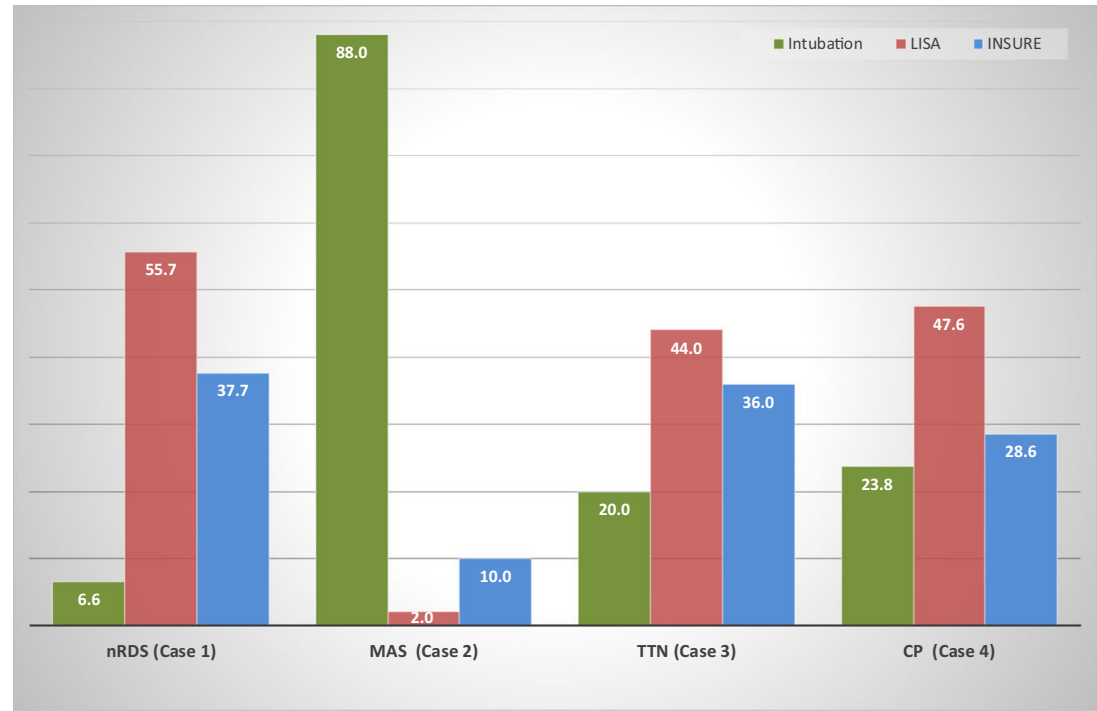


Fig. 3 LPT infant with nRDSage and $\mathrm{FiO}_{2}$ threshold for transfer to NICU. Expressed in \% of neonatologists. LPT, late preterm; nRDS, neonatal respiratory distress syndrome; NICU, neonatal intensive care unit

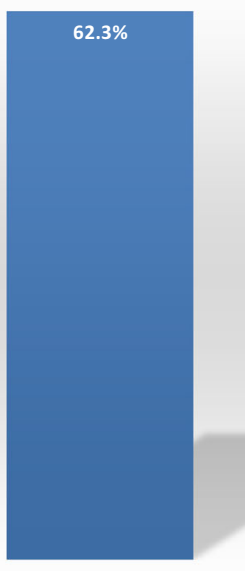

Fi02 $\geq 30 \%$ at the age of 12 hours

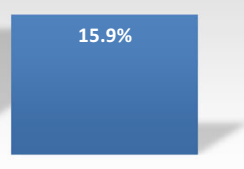

Fi02 $\geq 30 \%$ at the age of 24 hours

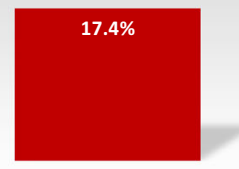

$\mathrm{FiO} 2 \geq 40 \%$ at the age of 12 hours

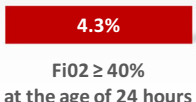

maximally $50 \mathrm{mg} / \mathrm{kg}$ between the recommended $(200 \mathrm{mg} / \mathrm{kg})$ and practical dosage $(150-200 \mathrm{mg} / \mathrm{kg})$. iNO is not seen as an alternative 'first line' treatment in this case.

In CP and MAS, those who will give surfactant dose at 200 $\mathrm{mg} / \mathrm{kg}$.

\section{Discussion}

When using surfactant for respiratory diseases in LPT infants, we observe a great deal of variability, both across Belgian NICU centres as well as among individual neonatologists. Such variability in care can be explained by a lack of evidence-based practice and may contribute to the currently observed morbidity among LPT infants. Using a survey on surfactant use in LPT infants across all Belgian neonatologists, a high response rate (86\%) was obtained which is significantly above a recommended $65 \%$ level [7]. Such makes this survey representative and thus less open to bias.

There are some limitations to creating a case-based survey. Firstly, as with any survey, data that are being produced are always likely to lack details or depth on the topic being investigated [7]. Secondly, at the outset of severe pulmonary disease in LPT infants the underlying aetiology is frequently unclear: respiratory disease often starts as a delayed respiratory transition and its subsequent course can be unpredictable. Thirdly, many LPT infants present mixed characteristics of nRDS, TTN and PPHN in the course of the same respiratory disease. In order to avoid ambiguous answers, we therefore opted to 'simplify' our survey using clearly delineated case presentations. A survey based on such well-described clinical cases is therefore somewhat different from the reality, as when confronted with an infant in respiratory distress, an exact diagnosis can only be obtained through the integration of anamnestic, clinical, imaging (especially lung ultrasound) and biological data (e.g. CRP). Such requires time and a significant effort, but it is the only way to understand what is the actual situation of that patient in that particular moment. This is particularly important as surfactant is expensive and may not always be effective. Fourthly, by looking at LPT infants (and thus a group of infants with a specific GA), we do not imply that surfactant administration should be based on GA. Clearly, the underlying pathophysiology of respiratory illnesses in these infants is a major reason why not all LPT infants may benefit from surfactant administration. Fifthly, a case-based survey remains a theoretical exercise on 'intended care', so caution is needed during the interpretation of answers. Finally, given the relatively small local newborn population (i.e. a total of approximately 6000 admissions annually across all Belgian NICUs), results need to be interpreted with caution. Indeed, some answers may have been influenced by the way neonatal health care is organised in Belgium and as such might be less relevant in different settings.

\section{Respiratory distress syndrome}

It is widely accepted that prophylactic use of surfactant has no advantage over the initiation of CPAP alone [8]. However, it remains important to determine when CPAP alone will not be effective. Whilst earlier studies recommend that surfactant should be administered as soon as $\mathrm{FiO}_{2}>0.30$ for very immature babies and $\mathrm{FiO}_{2}>0.40$ for more mature infants, the 2019 European directive recommends a threshold of $\mathrm{FiO}_{2}>$ 0.30 to be used for all infants with a clinical diagnosis of neonatal respiratory distress syndrome (nRDS) regardless of their GA [4]. The majority (76\%) of the Belgian neonatologists agrees with the principle that $\mathrm{FiO}_{2}>0.30$ in the first hours of life is a reasonable predictor of CPAP failure [9] and supports referral to a level III NICU as soon as $\mathrm{FiO}_{2}>0.30$ at the age of $12 \mathrm{~h}$. 
Intubation and ventilation are rarely used in nRDS (6.6\%), whilst such is more common in MAS, CP and severe TTN. A reduction of the percentage of invasive mechanical ventilation during the first 3 days of respiratory disease in LPT infants may thus be feasible in our country and should be aimed for, in line with the 2019 European directives [4].

\section{Transient tachypnoea of the neonate}

Whilst literature is scarce on surfactant administration within the clinical entity of transient tachypnoea of the newborn (TTN), it is somehow surprising to observe a fairly high percentage of neonatologists $(30.6 \%)$ is willing to administer surfactant in the case of TTN. Belgian neonatologists are not relying their final decision on chest X-ray appearance, but they rather take into account the level of $\mathrm{FiO}_{2}$. Some centres use pulmonary ultrasound, which is a promising bedside tool in order to differentiate between TTN and nRDS [10]. Pulmonary ultrasound has no adverse effects and allows neonatologists to detect those patients that may benefit from surfactant or mechanical ventilation, even prior to reaching oxygenation criteria $[11,12]$.

\section{Congenital pneumonia}

It is interesting to see that more than half of the neonatologists do not consider surfactant administration in congenital pneumonia (CP). From this group, $65 \%$ would still not administer surfactant if the $\mathrm{FiO}_{2}$ increases to 0.40 . Whilst the clinical picture of $\mathrm{nRDS}$ and TTN is very similar, CP is a clinically more heterogeneous entity, and probably therefore neonatologists are less inclined to consider the use of surfactant.

An interim analysis of a large ongoing international study suggests however that more and more neonatologists do consider surfactant in the case of CP [13] since CP often leads to surfactant deficiency or dysfunction $[14,15]$. However, in the absence of randomised controlled trials in LPT infants with proven or suspected pneumonia [16], one cannot state with certainty that any improvement in oxygenation upon surfactant therapy is either due to the surfactant itself or due to a natural recovery related to supportive treatment. Hence, the question of whether or not we should administer surfactant in $\mathrm{CP}$ remains unanswered, as reflected in our survey.

\section{Meconium aspiration syndrome}

In our survey, a significantly higher number of neonatologists opted to administer surfactant in meconium aspiration syndrome (MAS) compared with TTN and CP. The surfactant dysfunction in MAS seems to result in more from secondary inactivation than from primary deficiency [17]. Indeed, the aspiration of meconium induces pulmonary inflammation with subsequent type II cell dysfunction. Surfactant administration reduces the number of infants with progressive respiratory failure requiring support with ECMO $[18,19]$.

Our respondents state that surfactant in MAS should be given at high doses $(200 \mathrm{mg} / \mathrm{kg})$ and preferably as soon as possible when a trial of iNO with aggressive blood pressure management appears unsuccessful. Some studies suggest the opposite, i.e. that surfactant administration at a very early stage of MAS and prior to starting iNO may result in a better oxygenation due to optimised iNO diffusion [20]. Overall, the literature on MAS is more abundant than on CP [21]. The higher rate of surfactant administration observed in this survey reflects not only on the fact that patients with MAS are likely to present with a more severe respiratory failure than other disease groups but also on the fact that neonatologists base their decisions on available evidence.

\section{Congenital diaphragmatic hernia}

Pulmonary hypertension accounts for a significant morbidity and mortality in neonates with congenital diaphragmatic hernia $(\mathrm{CDH})$. Whether $\mathrm{CDH}$ is associated with alterations in alveolar surfactant composition and function remains controversial, as animal data are limited and sometimes difficult to confirm in clinical practice. For instance, antenatal treatment with vitamin A restores foetal rat lung maturation in an animal-model of $\mathrm{CDH}$, whilst most clinical studies have shown no significant benefit of vitamin A associated with surfactant therapy for (near) term infants with CDH [22]. There is however one study suggesting that partial alveolar SP-B deficiency could be a major contributor to respiratory insufficiency in newborn infants with $\mathrm{CDH}$ [23].

Notwithstanding this limited evidence for surfactant administration in $\mathrm{CDH}$, we were surprised to see that the majority of the respondents still use surfactant, predominantly as a rescue therapy post-surgery. $94 \%$ of the respondents administer $200 \mathrm{mg} / \mathrm{kg}$ even if the patient may have only one lung. Given the low incidence of CDH (1/4.000 infants $=30$ infants/ year in Belgium), exposure to this type of pathology is rare, and most likely, surfactant is being given as a 'last resort'. Nevertheless, if new-generation synthetic surfactantcontaining recombinant SP-B were available on the market, then it would be interesting to investigate whether alveolar SP-B correction might represent a therapeutic goal in $\mathrm{CDH}$ [23].

\section{Dosage issues during surfactant replacement therapy}

It is interesting to observe that a correct surfactant dosage of $200 \mathrm{mg} / \mathrm{kg}$ is not always followed; i.e. Belgian clinicians are tempted to administrate a rounded dose (to the vial content). As such, there exists a risk of under- and overtreating infants. For instance, a tendency to overtreat extremely low birth weight neonates and a trend to undertreat neonates $>28$ weeks 
GA has been reported in France and in Italy [24, 25]. A specific action to avoid under- or overtreating is to carefully weigh each infant prior to surfactant administration [26]. If however surfactant is required before the baby's birth weight is known (surfactant for early rescue therapy), it is acceptable to use whole vial dosing based on an estimated weight [9]. Undertreating small infants for financial reasons must be abandoned as such increases the odds for a retreatment with a second dose.

\section{Conclusion}

We demonstrated that a large fraction of Belgian neonatologists uses surfactant in LPT infants that present with pulmonary problems other than nRDS, such as MAS, TTN and CP. Respiratory care provided to this sizeable group of LPT infants remains an area with significant variability in practice. The high response rate to our survey initiative demonstrates a clear need to identify the best practice regarding surfactant therapy in LPT infants. Until large randomised controlled multi-centre trials on the use of surfactant in LPT infants are being organised, we will develop a national consensus statement, in order to reduce the current variability in respiratory care practice. Such qualityimprovement initiative may result in a more rational use of surfactant and possibly a reduction in strain on health care budgets.

Acknowledgements The authors would like to thank all neonatologists that participated in this study. Furthermore, they would like to thank the Bureau of the 'Belgian Association for Neonatologists' for their help in developing the questionnaire. Finally, they would like to thank Zarha Vermeulen, Nathalie Dirix and Pauline Capiau (Medical Department Chiesi) for providing logistic support during both Advisory Board meetings.

Authors' contributions All authors participated in the conceptualisation and design of the survey. LC carried out the data analysis and interpretation of the data and drafted the initial paper. All authors participated in discussing the survey (mini-Delphi technique). They also reviewed and revised the paper. All authors approved the final paper as submitted and agree to be accountable for all aspects of the work.

\section{Compliance with ethical standards}

Conflict of interest Chiesi provided logistic support (meeting room) for both advisory board meetings. All authors received honorarium from Chiesi as a reimbursement for travel costs. The authors maintained complete control over all content and phrasing within the manuscript and accept full responsibility for the content.

Ethical approval The survey has been approved by the Ethical Comite of AZ St-Jan Bruges-Ostend AV.
Informed consent Informed consent was obtained from all individual participants that completed the survey.

Disclaimer Neither Chiesi nor any other corporate entity contributed to data analysis/interpretation and preparation of the manuscript.

\section{References}

1. Escobar GJ, Clark RH, Greene JD (2006) Short-term outcomes of infants born at 35 and 36 weeks gestation: we need to ask more questions. Semin Perinatol 30(1):28-33

2. Boyle JD, Boyle EM (2013) Born just a few weeks early: does it matter? Arch Dis Child Fetal Neonatal Ed 98(1):F85-F88

3. Teune MJ, Bakhuizen S, Gyamfi Bannerman C et al (2011) A systematic review of severe morbidity in infants born late preterm. Am J Obstet Gynecol 205(4):374.e1-374.e9

4. Sweet DG, Carnielli V, Greisen G, Hallman M, Ozek E, te Pas A, Plavka R, Roehr CC, Saugstad OD, Simeoni U, Speer CP, Vento M, Visser GHA, Halliday HL (2019) European consensus guidelines on the management of respiratory distress syndrome - 2019 update. Neonatology. 115(4):432-450

5. Dani C, Mosca F, Vento G, Tagliabue P, Picone S, Lista G, Fanos V, Pratesi S, Boni L (2018) Effects of surfactant treatment in late preterm infants with respiratory distress syndrome. J Matern Fetal Neonatal Med 31(10):1259-1266

6. Bancalari EH, Jobe AH (2012) The respiratory course of extremely preterm infants: a dilemma for diagnosis and terminology. J Pediatr 161(4):585-588

7. Kelley K, Clark B, Brown V, Sitzia J (2003) Good practice in the conduct and reporting of survey research. Int J Qual Health Care 15(3):261-266

8. Isayama T, Chai-Adisaksopha C, McDonald SD (2015) Noninvasive ventilation with vs without early surfactant to prevent chronic lung disease in preterm infants: a systematic review and meta-analysis. JAMA Pediatr 169(8):731-739

9. Banerjee S, Fernandez R, Fox GF, Goss KCW, Mactier H, Reynolds P, Sweet DG, Roehr CC (2019) Surfactant replacement therapy for respiratory distress syndrome in preterm infants: United Kingdom national consensus. Pediatr Res 86(1):12-14

10. Brat R, Yousef N, Klifa R, Reynaud S, Shankar Aguilera S, de Luca D (2015 Aug) Lung ultrasonography score to evaluate oxygenation and surfactant need in neonates treated with continuous positive airway pressure. JAMA Pediatr 169(8):e151797

11. Raimondi F, Yousef N, Rodriguez Fanjul J, de Luca D, Corsini I, Shankar-Aguilera S, Dani C, di Guardo V, Lama S, Mosca F, Migliaro F, Sodano A, Vallone G, Capasso L (2019) A multicenter lung ultrasound study on transient tachypnea of the neonate. Neonatology. 115(3):263-268

12. Gregorio-Hernández R, Arriaga-Redondo M, Pérez-Pérez A, Ramos-Navarro C, Sánchez-Luna M (2020) Lung ultrasound in preterm infants with respiratory distress: experience in a neonatal intensive care unit. Eur J Pediatr 179(1):81-89

13. Crocker C, Courtney SE, van Kaam A, et al. The Neonatal ARDS Worldwide Network: summary on patient data on respiratory support, course and outcomes. Pediatr Acad Soc 2019 Meeting, abstract 3164737.

14. De Luca D, van Kaam AH, Tingay DG et al (2017) The Montreux definition of neonatal ARDS: biological and clinical background behind the description of a new entity. Lancet Respir Med 5(8): 657-666

15. Herting E, Gefeller O, Land M, van Sonderen L, Harms K, Robertson B, Members of the Collaborative European Multicenter Study Group (2000) Surfactant treatment of neonates 
with respiratory failure and group B streptococcal infection. Pediatrics. 106:957-964

16. Tan K, Lai NM, Sharma A (2012) Surfactant for bacterial pneumonia in late preterm and term infants. Cochrane Database Syst Rev 2: CD008155

17. Findlay RD, Taeusch HW, Walther FJ (1996) Surfactant replacement therapy for meconium aspiration syndrome. Pediatrics. 97(1): $48-52$

18. Lotze A, Knight GR, Martin GR, Bulas DI, Hull WM, O'Donnell RM, Whitsett JA, Short BL (1993) Improved pulmonary outcome after exogenous surfactant therapy for respiratory failure in term infants requiring extracorporeal membrane oxygenation. J Pediatr 122(2):261-268

19. Natarajan CK, Sankar MJ, Jain K, Agarwal R, Paul VK (2016) Surfactant therapy and antibiotics in neonates with meconium aspiration syndrome: a systematic review and meta-analysis. J Perinatol 36(Suppl 1):S49-S54

20. Rais-Bahrami K, Rivera O, Seale WR, Short BL (1997) Effect of nitric oxide in meconium aspiration syndrome after treatment with surfactant. Crit Care Med 25(10):1744-1747

21. El Shahed AI, Dargaville PA, Ohlsson A et al (2014) Surfactant for meconium aspiration syndrome in term and late preterm infants. Cochrane Database Syst Rev 12:CD002054

22. Lally KP, Lally PA, Langham MR, Hirschl R, Moya FR, Tibboel D, van Meurs K, Congenital Diaphragmatic Hernia Study Group
(2004) Congenital Diaphragmatic Hernia Study Group. Surfactant does not improve survival rate in preterm infants with congenital diaphragmatic hernia. J Pediatr Surg 39(6):829-833

23. Cogo PE, Simonato M, Danhaive O, Verlato G, Cobellis G, Savignoni F, Peca D, Baritussio A, Carnielli VP (2013) Impaired surfactant protein B synthesis in infants with congenital diaphragmatic hernia. Eur Respir J 41(3):677-682

24. Jourdain G, Zacaria F, Ammar F, De Luca D (2016) Appropriateness of surfactant dosing for preterm babies with respiratory distress syndrome: retrospective cohort study. Arch Dis Child Fetal Neonatal Ed 101(2):F182-F183

25. Boix H, Rite S, Arruza L, Fernandez C, Serrano I, Baquedano I, Sanchez A, Ferreira E, Fernandez P, Gonzalez R, Elorza MD (2019) Underdosing of surfactant for preterm babies with respiratory distress syndrome in clinical practice: a retrospective cohort study. Am J Perinatol 36(9):943-948

26. Foligno S, De Luca D (2020) Porcine versus bovine surfactant therapy for RDS in preterm neonates: pragmatic meta-analysis and review of physiopathological plausibility of the effects on extra-pulmonary outcomes. Respir Res 21(1):8

Publisher's note Springer Nature remains neutral with regard to jurisdictional claims in published maps and institutional affiliations. 